Agricultural Journal 7 (3): 194-197, 2012

ISSN: $1816-9155$

(C) Medwell Journals, 2012

\title{
Isolation, Sequence Identification and Tissue Expression Profile of a Novel Goat Gene $\mathrm{RHOA}$
}

\author{
Zhang Jing-Hu, Gong Wei-Hua and Yang Hong-Wen \\ Department of Biology Science and Technology, Zhangzhou Normal University, \\ Zhangzhou, 363000 Fujian, China
}

\begin{abstract}
The full-length cDNA sequence of one goat gene $R H O A$ was amplified using the rapid amplification of cDNA ends (RACE) method based on one goat EST sequence which was highly homologous to the coding sequence of human $R H O A$ gene. Sequence prediction analysis revealed that the open reading frame of this gene encodes a protein of 193 amino acids that shares high homology with the ras homolog gene family, member A (RHOA) of nine species human (100\%), cattle (100\%), sheep (100\%), rat (99\%), mouse ( $99 \%)$, chicken (99\%), dog $(99 \%)$, turkey (99\%) and green anole (99\%) so that it can be defined as goat RHOA gene. This novel goat gene was then deposited into NCBI database. Phylogenetic analysis revealed that the goat $R H O A$ gene has a closer genetic relationship with the $R H O A$ genes of human, cattle and sheep. Tissue expression analysis indicated that the goat $R H O A$ gene is generally and differentially expressed in detected tissues including spleen, muscle, skin, kidney, lung, liver, fat and heart. This experiment established the primary foundation for further research on the goat $R H O A$ gene.
\end{abstract}

Key words: Goat, RHOA, RACE, tissue expression profile, sequence identification, China

\section{INTRODUCTION}

Ras homolog gene family, member $\mathrm{A}(\mathrm{RHOA})$ is a member of RhoA-like subfamily. The RhoA subfamily consists of RHOA, RHOB and RHOC. RHOA promotes the formation of stress fibers and focal adhesions, regulating cell shape, attachment and motility (Su et al., 2006). RHOA is vital for muscle contraction for in vascular smooth muscle cells, RHOA plays a key role in cell contraction, differentiation, migration and proliferation (Peyton et al., 2008).

Recent researches demonstrated that RHOA is also involved in a lot of important biological processes such as GTP catabolic process, actin cytoskeleton organization, cell morphogenesis, cell-matrix adhesion, positive regulation of actin filament polymerization, negative regulation of neuron differentiation (Kobayashi et al., 2004; Beqaj et al., 2002; Gavard et al., 2004; Pixley et al., 2005; Ridley and Hall, 1992).

As mentioned before $R H O A$ gene is an important gene which has many biological functions. Until today, RHOA gene has been reported in cattle, human, mouse, rat and other animals. The goat $R H O A$ gene has not been reported. In the present experiment, researchers will clone the full-length cDNA sequence of goat $R H O A$ gene and further do necessary sequence analysis and tissue expression analysis. These will establish the primary foundation of understanding this goat gene.

\section{MATERIALS AND METHODS}

Animals and sample preparation: Two adult Fujian local goat were slaughtered. Spleen, muscle, skin, kidney, lung, liver, fat and heart samples were collected, frozen in liquid nitrogen and then stored at $-80^{\circ} \mathrm{C}$. The total RNA was extracted using the Total RNA Extraction Kit (Gibco, USA). These RNA samples were used to perform RACE PCR and tissue expression profile analysis.

5'- and 3'-RACE: About 5'- and 3'-RACE were performed to isolate the full-length $\mathrm{cDNA}$ for goat $R H O A$ gene as the instructions of SMART ${ }^{\mathrm{TM}}$ RACE cDNA Amplification Kit (Clontech, USA). For the goat RHOA gene, the GeneSpecific Primers (GSPs) were designed based on one goat EST sequence whose sequence is highly homologous to the coding sequence of human RHOA gene: EV435966. The Gene-Specific Primers (GSPs) were: 5'- RACE GSP: 5'CAAGGCACCCAGATTTTTTCTTCCC-3', 3'-RACE GSP: 5' - GAGGTTTTTGAAATGGCCACGAGAG. About -3' RACE touchdown PCRs were carried out with 5 cycles of

Corresponding Author: Zhang Jing-Hu, Department of Biology Science and Technology, Zhangzhou Normal University, Zhangzhou, 363000 Fujian, China 
$94^{\circ} \mathrm{C} / 30 \mathrm{sec}$ and $72^{\circ} \mathrm{C} / 3 \mathrm{~min}$, followed by 5 cycles of $94^{\circ} \mathrm{C}$ $/ 30 \mathrm{sec}, 70^{\circ} \mathrm{C} / 30 \mathrm{sec}$ and $72^{\circ} \mathrm{C} / 3 \mathrm{~min}$, finally with 30 cycles of $94^{\circ} \mathrm{C} / 30 \mathrm{sec}, 67^{\circ} \mathrm{C} / 30 \mathrm{sec}, 72^{\circ} \mathrm{C} / 3 \mathrm{~min}$ to terminate reaction. The RACE PCR products were then cloned into pMD18-T vector (TaKaRa, Dalian, China) and sequenced bidirectionally with the commercial fluorometric method.

RT-PCR for tissue expression profile analysis: RT-PCR for tissue expression profile analysis was performed as previously described elsewhere (Liu and Gao, 2009a, b; Liu, 2009). Researchers selected the housekeeping gene $\beta$-actin (Accession No.: AF481159) was performed as a positive control.

The $\beta$-actin gene primers used were: 5'TGGCATTGTCATGGACTC-3' (Forward primer 1) and 5'CCGTGGTGGTGAAGCT-3' (Reverse primer 1). The PCR product is $164 \mathrm{bp}$ in length. The following $R H O A$ gene specific primers were used to perform the RT-PCR for tissue expression profile analysis: 5' - AGGACCAGTT CCCAGAGG-3' (Forward primer 2) and 5'-AAGACAAGGC ACCCAGAT-3' (Reverse primer 2). The PCR product is 499 bp in length.

The $25 \mu \mathrm{L}$ reaction system was: $2 \mu \mathrm{L}$ cDNA (100 ng), 5 pmoles each oligonucleotide primer (forward and reverse primer 1 or forward and reverse primer 2), $2.5 \mu \mathrm{L} 2 \mathrm{mmol} \mathrm{L}^{-1}$ mixed dNTPs, $2.5 \mu \mathrm{L} 10 \times$ Taq DNA polymerase buffer, $2.5 \mu \mathrm{L} 25 \mathrm{mmol} \mathrm{L} \mathrm{MgCl}_{2}, 1.0$ units of Taq DNA polymerase and finally add sterile water to volume $25 \mu \mathrm{L}$. The PCR program initially started with a $94^{\circ} \mathrm{C}$ denaturation for $4 \mathrm{~min}$, followed by 30 cycles of $94^{\circ} \mathrm{C} / 50 \mathrm{sec}, 56^{\circ} \mathrm{C} / 50$ sec, $72^{\circ} \mathrm{C} / 50 \mathrm{sec}$ then $72^{\circ} \mathrm{C}$ extension for $10 \mathrm{~min}$, finally $4^{\circ} \mathrm{C}$ to terminate the reaction.

Sequence analysis: The cDNA sequence prediction was conducted using GenScan software. The protein prediction and analysis were performed using the Conserved Domain Architecture Retrieval Tool of BLAST at the National Center for Biotechnology Information (NCBI) server (http://www.ncbi.nlm.nih.gov/BLAST) and the ClustalW software (http://www.ebi.ac.uk/clustalw).

\section{RESULTS AND DISCUSSION}

RACE results for goat $R H O A$ gene: Through 5'-RACE, one PCR product of 797 bp was obtained. The 3'-RACE product was $709 \mathrm{bp}$. These products were then cloned to T-vector and sequenced. Taken together, a 1436-bp cDNA complete sequence was finally obtained (Fig. 1).

Sequence analysis: The nucleotide sequence analysis using the BLAST software at NCBI server (http://www.

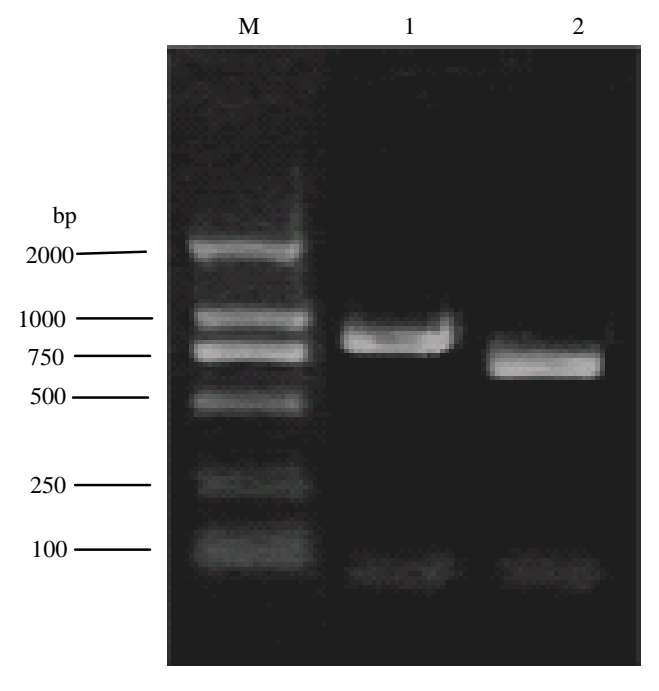

Fig. 1: RACE results for goat RHOA gene. M, DL2000 DNA markers 1, 5'-RACE product for $R H O A$ gene; 2, 3'-RACE product for $R H O A$ gene

ncbi.nlm.nih.gov/BLAST) revealed that this gene was not homologous to any of the known goat genes and it was then deposited into the GenBank database (Accession No.: JQ407045). The sequence prediction was carried out using the GenScan software. An open reading frame encoding 193 amino acids was found in the $1436 \mathrm{bp}$ cDNA sequence.

Further BLAST analysis of this protein revealed that this protein has high homology with the ras homolog gene family, member $\mathrm{A}(R H O A)$ of nine species human (Accession No.: NP_001655; 100\%), cattle (Accession No.: NP_788818; 100\%), sheep (Accession No.: NP_001155347; 100\%), rat (Accession No.: NP_476473, 99\%), mouse (Accession No.: NP_058082; 99\%), chicken (Accession No.: NP_990035; 99\%), dog (Accession No.: P24406; 99\%), turkey (Accession No.: ADX97247; 99\%) and green anole (Accession No.: XP_003217600; 99\%). The complete cDNA sequence of this gene and the encoded amino acids were shown in Fig. 2. From the sequencing and structural results described, this gene can be defined as the goat $R H O A$ gene.

Based on the results of the alignment of different species of RHOA (Fig. 3) a phylogenetic tree was constructed using the ClustalW software (http://www. ebi.ac.uk/clustalw) as shown in Fig. 4. The phylogenetic tree analysis revealed that the goat $R H O A$ gene has a closer genetic relationship with the $R H O A$ genes of human, cattle and sheep than with those of rat, mouse, chicken, dog, turkey and green anole.

Tissue expression profile: The RT-PCR analysis of the tissue expression profile was carried out using the tissue 
Agric. J., 7 (3): 194-197, 2012

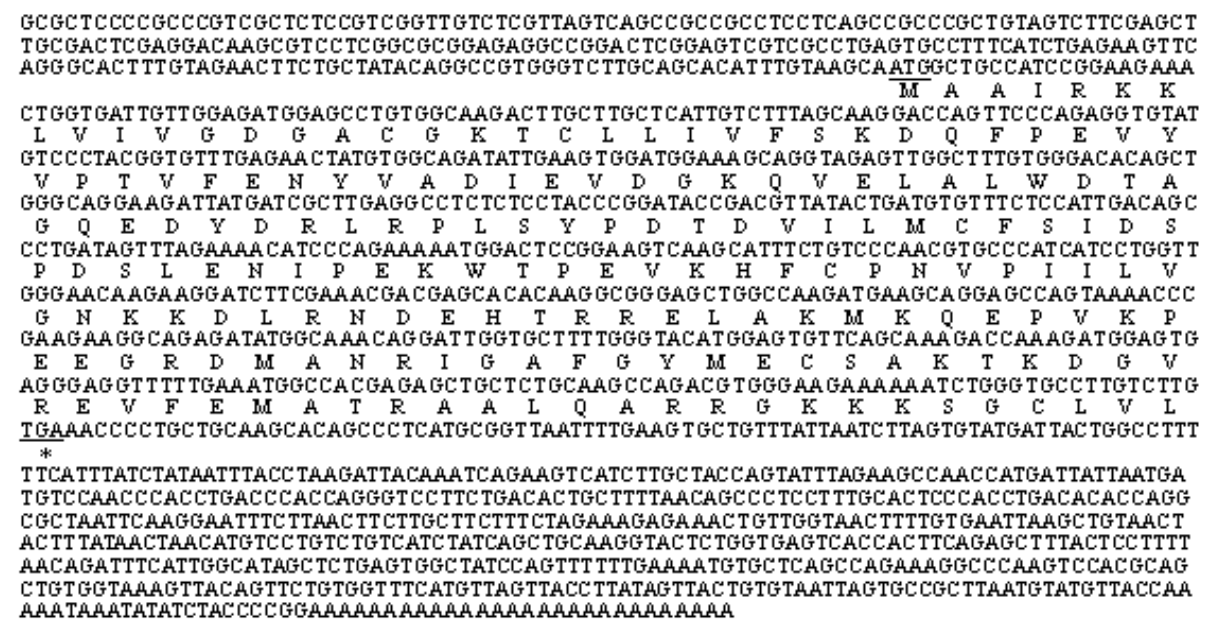

Fig. 2: The complete cDNA sequence and encoded amino acids of goat RHOA gene (GenBank Accession No: JQ407045). ATG, start codon; TAG, stop codon. *Indicates the stop codon

Turkey
Green anole
Chicken
Rat_Mouse
Goat_Human_Cattle_Sheep
Dog
Turkey
Green anole
Chicken
Rat_Mouse
Goat_Human_Cattle_Sheep
Dog
Turkey
Green anole
Chicken
Rat_Mouse
Goat_Human_Cattle_Sheep
Dog

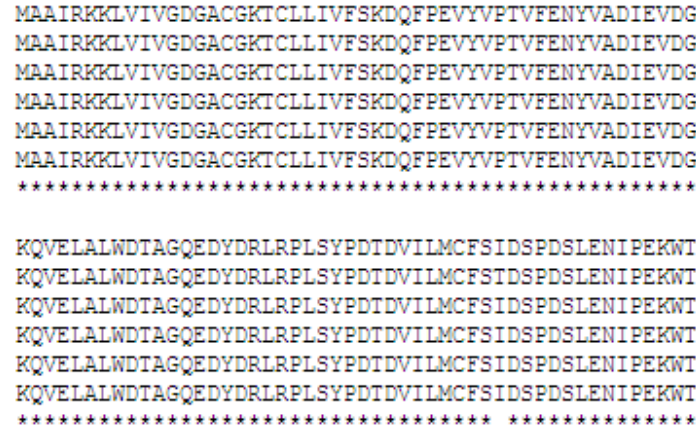

PEVKHFCPNVPI ILVGNKKDLRNDEHTRRELAKMIKQE PVKPEEGRDMANR PEVKHFCPNVPIILVGNKKDLRNDEHTRRELAKMKQE PVKPEEGRDMANR PEVKHFCPNVPIILVGIKKDLRNDEHTRRELAKMKOE PVKPEEGRDMANR PEVKHFCPNVPIILVGIKKDLRNDEHTRRELAKMKQEPVKPEEGRDMANR PEVKHFCPNVPI ILVGNKKDLRNDEHTRRELAKMKQE PVKPEEGRDMANR PEVKHFCPNVPIILVGIKKKDLRNDEHTRRELAKMIKQEPVKPTEGRDMAIR

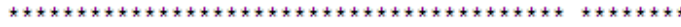

IGAFGYMECSAKTKDGVREVFEMATRAALQARRGRKKSGCLLI IGAFGYMECSAKTKDGVREVFEMATRAALQARRGKKKSGCLLI IGAFGYMECSAKTKDGVREVFEMATRA_LLARRGKKKSGCLLI IGAFGYMECSAKTKDGVREVFEMATRAALQARRGKKKSGCLIL IGAFGYMECSAKTKDGVREVFEMATRAALQARRGKKKSGCLVL IGAFGYMECSAKTKDGVREVFEMATRAALOARRGKKKSGCLVL

Fig. 3: The alignment of the protein encoded by $R H O A$ gene from goat and nine other kinds of RHOA proteins from human, cattle, sheep, rat, mouse, chicken, dog, turkey and green anole

cDNAs as the templates and results indicated that the goat $R H O A$ gene is generally and differentially expressed in detected tissues including spleen, muscle, skin, kidney, lung, liver, fat and heart (Fig. 5).

Through sequence analysis, researchers found that the encoding protein of goat $R H O A$ gene is highly homologous with RHOA proteins of human, mouse and other mammals. This implied that the $R H O A$ genes were highly conserved in some mammals and the goat $R H O A$ gene will have similar functions as the $R H O A$ genes of human, mouse and other mammals. Researcher also found that the goat RHOA protein does not show complete identity to mouse or other mammals.

This implied that goat $R H O A$ gene will have some differences in functions to those $R H O A$ genes from mouse or other mammals. From phylogenetic analysis, 
Agric. J., 7 (3): 194-197, 2012

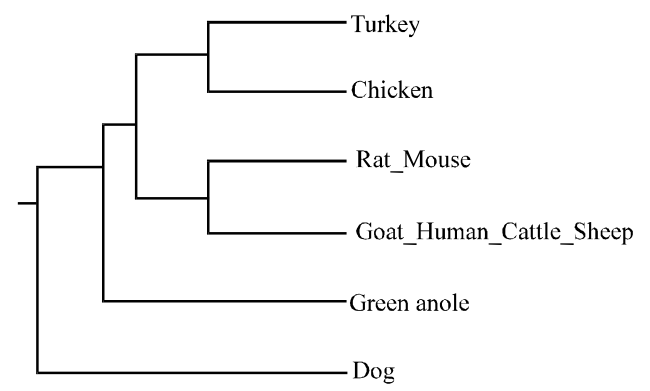

Fig. 4: The phylogenetic tree for ten kinds of RHOA from goat, human, cattle, sheep, rat, mouse, chicken, dog, turkey and green anole

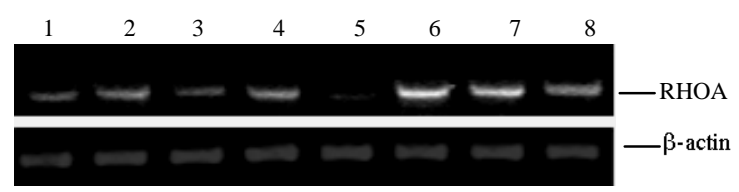

Fig. 5: Tissue expression profile analysis of the goat RHOA gene on the agarose gel of $1 \%$ stained with ethidium bromide. The $\beta$-actin expression is the control (1, skin; 2 , kidney; 3 , spleen; 4 , heart; 5 , fat; 6 , lung; 7 , liver; 8 , muscle)

researchers found that goat $R H O A$ gene has a closer genetic relationship with the $R H O A$ genes of human, cattle and sheep, this implied that we can use human, cattle and sheep as model organisms to study the goat RHOA gene. From the tissue distribution analysis in the research, it can be seen that the goat $R H O A$ gene was obviously differentially expressed in some tissues. As researcher did not study functions at protein levels yet, there might be many possible reasons for differential expression of goat $R H O A$ gene. The suitable explanation for this under current conditions is that at the same time those biological activities related to the mRNA expression of goat $R H O A$ gene were presented diversely in different tissues.

\section{CONCLUSION}

In this study, researchers first isolated the goat RHOA gene and performed necessary sequence analysis and tissue transcription profile analysis. This established the primary foundation for further insight into this novel goat gene.

\section{REFERENCES}

Beqaj, S., S. Jakkaraju, R.R. Mattingly, D. Pan and L. Schuger, 2002. High RhoA activity maintains the undifferentiated mesenchymal cell phenotype, whereas RhoA down-regulation by laminin-2 induces smooth muscle myogenesis. J. Cell. Biol., 156: 893-903.

Gavard, J., M. Lambert, I. Grosheva, V. Marthiens and T. Irinopoulou et al., 2004. Lamellipodium extension and cadherin adhesion: Two cell responses to cadherin activation relying on distinct signalling pathways. J. Cell Sci., 117: 257-270.

Kobayashi, K., M. Takahashi, N. Matsushita, J.I. Miyazaki and M. Koike et al., 2004. Survival of developing motor neurons mediated by Rho GTPase signaling pathway through Rho-kinase. J. Neurosci., 24: $3480-3488$.

Liu, G.Y., 2009. A novel HADHA gene differentially expressed in muscle and other tissues from blackboned vs. ordinary sheep. Anim. Sci. Pap. Rep., 27: 127-137.

Liu, Y.G. and S.Z. Gao, 2009a. A novel goat gene, MMP7, differentially expressed in muscles from black-boned sheep and local common sheep. J. Applied Genet., 50: 253-256.

Liu, Y.G. and S.Z. Gao, 2009b. Molecular cloning, sequence identification and tissue expression profile of three Novel sheep (Ovis aries) Genes - BCKDHA, NAGA and HEXA. Biol. Res., 42: 69-77.

Peyton, S.R., P.D. Kim, C.M. Ghajar, D. Seliktar and A.J. Putnam, 2008. The effects of matrix stiffness and RhoA on the phenotypic plasticity of smooth muscle cells in a 3-D biosynthetic hydrogel system. Biomaterials, 29: 2597-2607.

Pixley, F.J., Y. Xiong, R.Y. Yu, E.A. Sahai, E.R. Stanley, B.H. Ye, 2005. BCL6 suppresses RhoA activity to alter macrophage morphology and motility. J. Cell Sci., 1: 1873-1883.

Ridley, A.J. and A. Hall, 1992. The small GTP-binding protein tho regulates the assembly of focal adhesions and actin stress fibers in response to growth factors. Cell, 70: 389-399.

$\mathrm{Su}$, W., P. Chardin, M. Yamazaki, Y. Kanaho and G. Du, 2006. RhoA-mediated phospholipase D1 signaling is not required for the formation of stress fibers and focal adhesions. Cell Signal, 18: 469-478. 